\title{
JORDAN OPERATORS IN INFINITE DIMENSIONS AND STURM LIOUVILLE CONJUGATE POINT THEORY ${ }^{1}$
}

\author{
BY J. WILLIAM HELTON \\ Communicated by P. D. Lax, July 21, 1971
}

This note concerns the two simplest types of bounded operators with real spectrum on a Hilbert space $H$. The purpose of this note is to suggest an abstract algebraic characterization for these operators and to point out a rather unexpected connection between such algebraic considerations and the classical theory of ordinary differential equations. In particular, our Theorem II which gives an algebraic characterization of certain subjordan operators (defined below) seems very closely related to the classical theorem asserting that a Sturm Liouville operator defined on the interval $[a, b]$ is positive definite if and only if there are no points conjugate to $a$ in the interval. One appealing thing is that almost every idea presented here has a natural generalization worthy of investigation.

The two types of operators considered here are:

Jordan operators (order $k$ )-operators of the form $S+N$ where $S$ is selfadjoint, $S$ commutes with $N$, and $N^{k}=0$.

Subjordan operators (order $k$ )-operators which are unitarily equivalent to the restriction of a Jordan operator $C$ to an invariant subspace of $C$.

A natural algebraic condition on a bounded operator $T$ which generalizes the selfadjointness condition is

POL $n$

$$
e^{-i s T^{*}} e^{i s T}=\sum_{k=0}^{n} A_{k} s^{k}
$$

This is equivalent to

$$
\frac{d^{n+1}}{d s^{n+1}} e^{-i s T^{*}} e^{i s T}=0
$$

which is in turn equivalent to $C_{T}^{n+1}(I)=0$, where $C_{T}: \mathscr{L}(H) \rightarrow \mathscr{L}(H)$ is defined by $C_{T}(A)=T^{*} A-A T$ and $C_{T}^{k}$ denotes the composition of the map $C_{T}$ with itself $k$ times. An operator $T$ which satisfies POL $n$ will be called coadjoint (order $n$ ). Note that if $T$ is coadjoint, then $T^{*}$ is not necessarily coadjoint. The results announced here concern coadjoint operators of the second order only. Details of proofs will appear elsewhere.

THEOREM I. An operator T is Jordan (order 2) if and only if both T and $T^{*}$ are coadjoint (order 2).

AMS 1969 subject classifications. Primary 4730, 4740, 3442; Secondary 4760.

${ }^{1}$ This research was performed while the author was partially supported by NSF Grant GP-19587. 
Next we turn to subjordan operators. It is obvious that any subjordan operator satisfies the POL condition of the appropriate order and it seems natural to ask

Question. When is a coadjoint operator a subjordan operator?

The purpose of this note is to give an idea of what this question involves by outlining the proof of the following statement.

THEOREM II. If $T$ is coadjoint (order 2 ), if $T$ has a cyclic vector $\psi_{0}$, if spectrum $T=[a, b]$, and if $T$ satisfies a strong additional technical assumption (to be described later), then $T$ is a subjordan operator (order 2).

Of interest in its own right is the generalization of the selfadjoint spectral theorem.

THEOREM III. If $T$ is a coadjoint operator, if $T$ has a cyclic vector, and if spectrum $T=[a, b]$, then $T$ is unitarily equivalent to "multiplication by $x$ " on a sort of generalized Sobolev space supported on $[a, b]$.

For more on this last theorem see Part I in the outline for Theorem II.

Now we introduce the notions needed to describe the technical assumption in Theorem II. Henceforth assume that $T$ satisfies POL 2. It is clear from POL 2 that

$$
\frac{e^{-i s T^{*}} e^{i s T}}{s^{2}} \rightarrow A_{2} .
$$

This implies two things: first $A_{2}$ is a nonnegative operator, and second $e^{i s T^{*}} A_{2} e^{-i s T}=A_{2}$ for each $s$. If $A_{2}$ has no null vectors, then $[x, y]_{2}$ $=\left[A_{2} x, y\right]$ is a positive definite bilinear form on $H([$,$] denotes the inner$ product on $H$ ) and can be completed to a Hilbert space $\mathrm{H}_{2}$ which contains $H$ as a dense subspace. The relation $e^{i s T^{*}} A_{2} e^{-i s T}=A_{2}$ is equivalent to the statement that $e^{i s T}$ is a one parameter unitary group on $H_{2}$. The infinitesimal generator $T$ of this group is selfadjoint and consequently can be thoroughly studied as an operator on $\mathrm{H}_{2}$ by using its spectral resolution which we write $T=\int \lambda d E_{\lambda}$. Let $l$ stand for Lebesgue measure and take $[a, b]=[0,1]$ henceforth.

TECHNICAL ASSUMPTION (IN THEOREM II). There are positive numbers $M$ and $m$ so that, for any subset $S$ of $[0,1], m l(S) \leqq\left[A_{2} E(S) \psi_{0}, \psi_{0}\right] \leqq M l(S)$ where $\psi_{0}$ is the cyclic vector for $T$ mentioned in Theorem II.

It turns out that this assumption is invariant under change of cyclic vector. Now we sketch the idea behind Theorems I and II. The proof of Theorem $I$ is purely algebraic. One verifies 5 or 6 identities about $T, C_{T}(I)$ $=-C_{T^{*}}(I), C_{T}^{2}(I)$, and $C_{T^{*}}^{2}(I)$ and uses them to show that the operator $N=i A_{1}^{-1} A_{2}$ is a legitimate bounded operator with square 0 , and with the properties $C_{N}(I)=C_{T}(I), C_{N}^{2}(I)=C_{T}^{2}(I)$, and $C_{N^{*}}^{2}(I)=C_{T^{*}}^{2}(I)$. With a good 
bit more manipulation it can be shown that $T-N=S$ is selfadjoint and that $S$ commutes with $N$.

The proof of Theorem II relies on analysis and sits within the framework of the study of operators with a $C^{n}$-functional calculus (cf. [C-F]). We break it into 4 parts.

Part I. A representation for coadjoint operators. Let $\mathscr{S}\left(R^{n}\right)$ denote the Schwartz space of $C^{\infty}$ functions whose derivatives decrease faster than any polynomial at infinity. Define

$$
\varphi(f)=\frac{1}{\sqrt{2 \pi}} \int_{-\infty}^{\infty} \hat{f}(s) e^{-i s T} d s \quad \text { for } f \in \mathscr{S}\left(R^{1}\right) .
$$

The map $\varphi$ is a continuous algebraic homomorphism of $\mathscr{S}\left(R^{1}\right)$ into $L(H)$. By the Schwartz Nuclear Theorem, there is a distribution $b$ on $\mathscr{S}\left(R^{2}\right)$, the Schwartz space on $R^{2}$, such that $b(f(x) \overline{g(y)})=\left[\varphi(f) \psi_{0}, \varphi(g) \psi_{0}\right]$ for each $f, g \in \mathscr{S}$. The POL $n$ condition is equivalent to

$$
0=\frac{d^{n+1}}{d s^{n+1}} b\left(e^{i s(x-y)} f(x) \overline{g(y)}\right)=b\left([x-y]^{n+1} f(x) \overline{g(y)}\right)
$$

which implies that the distribution $b$ has support on the line $\{(x, x)\}$. From this, one can apply the Gelfand representation for the Banach algebra of operators of the form $\varphi(f)$, and get that $\Delta=\{(x, x): x \in \sigma(T)=[0,1]\}$ is the support of $b$. The usual representation for distributions (Schwartz Kernel Theorem) allows us to write $b$ as

$$
b(h(x, y))=\sum_{i, j=0}^{M} \int_{0}^{1} K_{i, j}(x, x) \frac{\partial^{i+j}}{d x^{i} d y^{j}} h(x, x) d x
$$

for some $K_{i j}$. For the details of the preceding argument see [H]. After quite a lot of work the fact that $\left[\varphi(f) \psi_{0}, \varphi(g) \psi_{0}\right]$ is a positive bilinear form gives us that $M=1$ in (1) and consequently

$$
\begin{aligned}
& {\left[\varphi(f) \psi_{0}, \varphi(g) \psi_{0}\right]} \\
& \quad \operatorname{REP} \quad=\int_{0}^{1} f \bar{g} d \mu_{00}+\int_{0}^{1} f^{\prime} \bar{g} d \mu_{10}+\int_{0}^{1} f \bar{g}^{\prime} d \mu_{01}+\int_{0}^{1} f^{\prime} \bar{g}^{\prime} d \mu_{11} \\
& \quad \equiv(f, g)_{1}
\end{aligned}
$$

where the $\mu_{i j}$ are finite signed Borel measures, $\mu_{10}=\bar{\mu}_{01}, \mu_{00}=\bar{\mu}_{00}$ and $\mu_{11}$ is a positive measure. Let $\boldsymbol{\mu}$ denote the matrix

$$
\left(\begin{array}{ll}
\mu_{00} & \mu_{01} \\
\mu_{10} & \mu_{11}
\end{array}\right)
$$

of measures and let $H(\mu)$ denote the closure of the $C^{\prime}[0,1]$ functions (once 
differentiable) in $(,)_{1} . H(\mu)$ is a sort of generalized Sobolev space and $T$ on $H$ is unitarily equivalent to "multiplication by $x$ " on $H(\boldsymbol{\mu})$.

Part II. The problem reduces to one of positive definiteness. Define a bilinear form \{\}$_{\mu}$ on the 2 -vector valued $C^{\prime}[0,1]$ functions (once differentiable) by

$$
\left\{\left(\begin{array}{l}
f_{0} \\
f_{1}
\end{array}\right)\left(\begin{array}{l}
g_{0} \\
g_{1}
\end{array}\right)\right\}=\sum_{i, j=0}^{1} \int_{0}^{1} f_{i} g_{j} d \mu_{i j} .
$$

Suppose that $\{,\}_{\mu}$ is a positive definite bilinear form and denote by $\mathscr{H}(\boldsymbol{\mu})$ the completion of the 2-vector valued $C^{\prime}[0,1]$ functions in $\{,\}_{\mu}$. Let $\mathscr{R}$ denote the functions in $\mathscr{H}(\boldsymbol{\mu})$ of the form $\left({ }_{f^{\prime}}\right)$. Clearly the map $\tau: H(\boldsymbol{\mu}) \rightarrow \mathscr{R}$ given by $\tau(f)=\left(f_{f}^{\prime}\right)$ is an isometry of $H(\boldsymbol{\mu})$ onto $\mathscr{R}$. Moreover, the map $f \rightarrow x f$ on $H(\mu)$ induces (under $\tau$ ) the map

$$
\left(\begin{array}{l}
f \\
f^{\prime}
\end{array}\right) \underset{T}{\rightarrow}\left(\begin{array}{ll}
x & 0 \\
1 & x
\end{array}\right)\left(\begin{array}{l}
f \\
f^{\prime}
\end{array}\right) \quad \text { on } \mathscr{R} .
$$

The operator $J$ given by

$$
J\left(\begin{array}{l}
f_{0} \\
f_{1}
\end{array}\right)=\left(\begin{array}{ll}
x & 0 \\
1 & x
\end{array}\right)\left(\begin{array}{l}
f_{0} \\
f_{1}
\end{array}\right) \quad \text { on } \mathscr{H}(\boldsymbol{\mu})
$$

is clearly Jordan and $\tilde{T}$ is $J$ restricted to $\mathscr{R}$. Thus the original Hilbert space $H$ is unitarily equivalent to $\mathscr{R}$ and the operator $T$ on $H$ under this unitary map becomes $J$ restricted to $\mathscr{R}$. Thus Theorem II has been reduced to showing that \{\}$_{\mu}$ is positive definite.

Part III. A simplified representation. Now we invoke the Technical Assumption of Theorem II. It says precisely that $d \mu_{11}=\tilde{P}(\lambda) d \lambda$ where $\tilde{P}$ is a function with the bounds $m \leqq \tilde{P}(\lambda) \leqq M$. This assumption along with the positivity of $(,)_{1}$ forces $d \mu_{10}$ to have the form $d \mu_{10}=R(\lambda) d \lambda$ where $R$ is a Lebesgue $L^{2}$ function. Now we change cyclic vector. The old cyclic vector $\psi_{0}$ corresponds to the function 1 ; pick the new cyclic vector $\psi_{c}$ to correspond to the function $c(x)=\exp \left\{-\int_{0}^{x} R / \tilde{P}\right\}$. Then

$$
\left[\varphi(f) \psi_{c}, \varphi(g) \psi_{c}\right]=\int_{0}^{1} f \bar{g} d v_{00}+\int_{0}^{1} f^{\prime} \bar{g}^{\prime} P d \lambda \equiv(f, g)_{c}
$$

where $v_{00}$ is a finite signed Borel measure. The norm $\sqrt{ }(,)_{c}$ is equivalent to the Sobolev space $H_{1}$ norm, $\|f\|^{1}=\int_{0}^{1}\left|f^{\prime}\right|^{2} d x+\int_{0}^{1}|f|^{2} d x$.

Part IV. The Jacobi conjugate point theorem. If $d v_{00}=Q(\lambda) d \lambda$ and if $P$ were differentiable $(f, g)_{c}$ could be written $\int_{0}^{1}[L f] \bar{g} d \lambda+\left.P f^{\prime} g\right|_{0} ^{1}$ where $L f=-\left(P f^{\prime}\right)^{1}-Q f$, and a considerable body of Sturm Liouville theory would apply. For the sake of exposition we shall assume that $d v_{00}$ $=Q(\lambda) d \lambda$, cite what is needed from Sturm Liouville theory, and assure the 
reader that standard techniques can be modified to handle the case at hand. Using integration by parts one can write

$$
(f, g)_{c}=\int_{0}^{1} f \bar{g}\left[Q+B^{\prime}\right] d x+\int_{0}^{1}(f \bar{g})^{\prime} B d x+\int_{0}^{1} f^{\prime} \bar{g}^{\prime} P-\left.f \bar{g} B\right|_{0} ^{1}
$$

where $B$ is any real differentiable function. If we ignore boundary terms, the matrix of measures associated with this representation for $(,)_{c}$ is $\mu_{B}=\left({ }_{B}^{+B^{\prime}}{ }_{P}^{B}\right) d x$.

Part II tells us that (modulo boundary terms) the proof is complete if we can find a function $B$ for which $\operatorname{det} \mu_{B}=\left(Q+B^{\prime}\right) P-B^{2}>0$ pointwise on $[0,1]$. Make the usual substitution $B=-P w^{\prime} / w$ and observe that

$$
\left(Q+B^{\prime}\right) P=B^{2}
$$

when $w$ satisfies $L w=0$. Thus if a nonvanishing solution $w$ to $L w=0$ exists, a solution $B$ to (2) exists. The preceding argument is tantamount to a proof of one side of the classical theorem (see [D-S], XIII. 7).

THEOREM. The Sturm Liouville operator L on $[0,1]$ with certain selfadjoint boundary conditions is positive definite if and only if there is a solution $w$ to $L w=0$ which is positive on $[0,1]$.

Now we can use the reverse side of this theorem to conclude from the fact that $(f, g)_{c}$ is positive definite that there is a solution $w$ of $L w=0$ which is positive on $[0,1]$. Thus we are done except for the boundary terms which were ignored and the fact that what we wanted was not $\left(Q+B^{\prime}\right) P=B^{2}$ but strict inequality. One can use a continuous dependence argument to get strict inequality and the fact that $(f, f)_{c}$ is positive for all $f$ in $C^{\prime}[0,1]$ is precisely what is needed to take care of the boundary conditions.

\section{BIBLIOGRAPHY}

[C-F] I. Colojara and C. Foias, Theory of generalized spectral operators, Gordon and Breach, New York, 1968.

[D-S] N. Dunford and J. T. Schwartz, Linear operators. II: Spectral theory. Selfadjoint operators in Hilbert space, Interscience, New York, 1963. MR 32 \#6181.

$[\mathbf{H}]$ J. William Helton, Operators with a representation as 'multiplication by $x$ ' on a Sobolev space, Proc. Internat. Conference on Operator Theory, Hungary, 1970 (to appear).

Department of Mathematics, State University of New York, Stony Brook, New YORK 11970 